\title{
Scarring in a driven system with wave chaos
}

\author{
A.L. Virovlyansky \\ Institute of Applied Physics, 46 Ulyanov St., Nizhny Novgorod 603950, Russia \\ G.M. Zaslavsky \\ Courant Institute of Mathematical Sciences, New York University 251 Mercer St., \\ New York, NY 10012 and Department of Physics, \\ New York University 2-4 Washington Place, New York, NY 10003, USA
}

\begin{abstract}
We consider acoustic wave propagation in a model of a deep ocean acoustic waveguide with a periodic range-dependence. Formally, the wave field is described by the Schrödinger equation with a time-dependent Hamiltonian. Using methods borrowed from the quantum chaos theory it is shown that in the driven system under consideration there exists a "scarring" effect similar to that observed in autonomous quantum systems.
\end{abstract}

It is well-known in the theory of quantum chaos that classical periodic orbits, to a significant extent, determine both the distribution of energy levels and structures of eigenfunctions 1, 2, 3]. In particular, for some eigenfunctions, the amplitudes are high in the vicinity of unstable periodic orbits and low elsewhere. This phenomenon, first discovered in a quantum billiard, is called the scarring [4]. It has been established that the scarring is a common property for many autonomous chaotic systems $[3,[5,[6]$. The description of scarring is related to the problem of construction of eigenfunctions on the basis of purely semiclassical calculations $\mathbf{7}, 8,0,10,11,12$.

It is also well-known that so different physical problems as quantum particle dynamics and wave propagation in nonuniform media in the narrow beam approximation are described by the same parabolic (or Shrödinger) equation. One of the most intriguing object is the underwater acoustic (UWA) and the long range sound propagation in the ocean 13, 14, 15]. Application of methods of quantum chaos to the wave chaos proves to be efficient in many publications 16, 17, 18, 19, 20. The goal of this paper is to extend this analogy between quantum and wave chaos and to present simulations that demonstrate the phenomenon of scarring in a nonautonomous Hamiltonian system with 1.5 degrees of freedom related to the UWA. We consider a simplified case of wave propagation in a two dimensional range-dependent model of hydroacoustic waveguide that has been used to study wave and ray chaos at long range sound propagation in the ocean [21, 22, 23, 24, 25]. Formally, the simplified model of ray propagation is equivalent to a nonlinear oscillator perturbed by a periodic force, while the corresponding parabolic equation is equivalent to the Shrödinger equation for the oscillator.

Consider a two-dimensional UWA waveguide with the sound speed $c$ as a function of depth, $z$, and range, $r$. In the parabolic equation approximation (valid under assumptions that waves propagate at small grazing angles) the monochromatic wave field $u$ at a carrier frequency $f$ obeys the parabolic equation [26, 27]

$$
\frac{i}{k} \frac{\partial u}{\partial r}=\hat{H} u, \hat{H}=-\frac{1}{2 k^{2}} \frac{\partial^{2}}{\partial z^{2}}+U(r, z),
$$

where $U(r, z)=\left(1-\bar{c}^{2} / c^{2}(r, z)\right) / 2$. Here $\bar{c}$ and $k=$ $2 \pi f / \bar{c}$ are a reference sound speed and a reference wavenumber, respectively. Equation (1) formally coincides with the time-dependent Schrödinger equation. In this analogy $r$ plays a role of time, $\hat{H}$ is the Hamiltonian operator, $U(r, z)$ is the potential, and $k^{-1}$ associates with the Planck constant. The ray paths are determined by the standard Hamilton equations $d p / d t=$ $-\partial H / \partial z$ and $d z / d r=\partial H / \partial p$ with the Hamiltonian $H=p^{2} / 2+U(r, z)$, where the momentum $p$ is related to the ray grazing angle $\theta$ by $p=\tan \theta$.

Following Ref. 21] we take the sound speed field $c(r, z)=c_{0}(z)+\delta c(r, z)$, where $c_{0}(z)$ is the so-called Munk profile widely used to model sound propagation in the deep sea [27],

$$
c_{0}(z)=\bar{c}\left(1+\varepsilon\left(\left(e^{-2\left(z-z_{a}\right) / B}+2\left(z-z_{a}\right) / B-1\right)\right)\right.
$$

with $\bar{c}=1.5 \mathrm{~km} / \mathrm{s}, z_{a}=-1 \mathrm{~km}, \varepsilon=0.0057$, and $B=1$ $\mathrm{km}$, and

$$
\delta c(r, z)=-2 \gamma \bar{c} z / B e^{-2 z / B} \sin (2 \pi r / L) .
$$

with $\gamma=0.01$ and $L=10 \mathrm{~km}$ is a perturbation that generates the ray chaos. The corresponding potential $U(r, z)$ is a periodic function of range. Its shapes at three different ranges are shown in Fig. 1.

Figure 2 presents the phase portrait of the ray system plotted using the Poincaré map [16, 17, 21, 28, 29] $\left(p_{n+1}, z_{n+1}\right)=\hat{T}\left(p_{n}, z_{n}\right)$, where $\hat{T}$ denotes transformation of the momentum and coordinate of a ray trajectory taken at range $n L$ to range $(n+1) L$ for $n=0,1, \ldots$ We see a typical picture of stable islands formed by regular curves submerged in a chaotic sea filled with randomly scattered points depicting chaotic rays.

To find out how the structure in Fig. 2 reveals itself in a wave dynamics described by Eq. (1) we shall use 


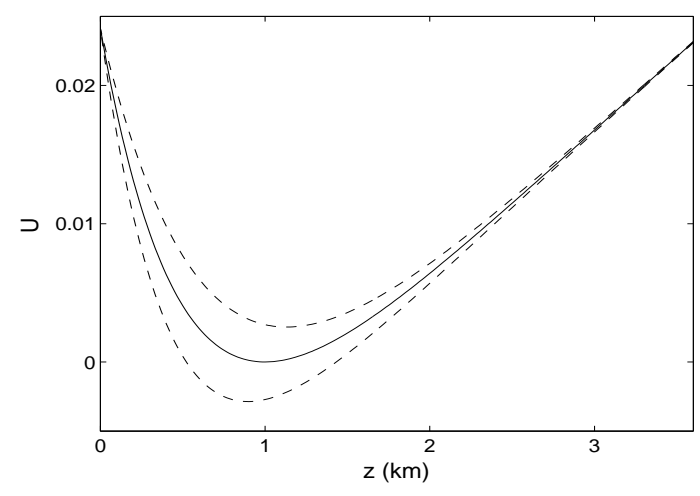

FIG. 1: Unperturbed potential $U(0, z)$ (solid line) and its maximal deviations (dashed line) due to the perturbation.



FIG. 2: Phase portrait of the ray system.

the Floquet theorem [2, 3] and follow Ref. [30] where a similar approach was applied. The solution of Eq. (11) can be presented as a sum of Floquet modes

$$
u_{m}(r, z)=e^{i \alpha_{m} r / L} \Psi_{m}(r, z),
$$

where $\Psi_{m}(r+L, z)=\Psi_{m}(r, z)$ are range-periodic, $\alpha_{m}$ are real constants, and $m=0,1, \ldots$ In quantum mechanics analogous terms describe the so-called Floquet states, and constants $\alpha_{m}$ are proportional to the quasienergies. $\Psi_{m}(r, z)$ are eigenfunctions of the shift operator $\hat{F}: \hat{F} \Psi_{m}(r, z)=\Psi_{m}(r+L, z)=e^{i \alpha_{m}} \Psi_{m}(r, z)$. Since $\hat{F}$ is Hermitian, $\Psi_{m}(r, z)$ form a complete set [2, 3].

Let us consider functions $\psi_{m}(z) \equiv \Psi_{m}(0, z)$. They can be presented as a decomposition $\psi_{m}(z)=\sum_{q} C_{m q} \varphi_{q}(z)$, where $\varphi_{q}(z)(q=0,1, \ldots)$ are solutions to SturmLiouville eigenvalue problem $\hat{H}_{0} \varphi_{m}=E_{m} \varphi_{m}(z)$ in the unperturbed waveguide with the Hamiltonian $\hat{H}_{0}$ corresponding to $c(r, z)=c_{0}(z)$. The eigenfunctions $\varphi_{m}(z)$ and eigenvalues $E_{m}$ are readily found using a standard mode code [31]. It is easy to see that the phase factors $e^{i \alpha_{m}}$ and columns of matrix $\left\|C_{m q}\right\|$ are eigenvalues and eigenvectors, respectively, of a unitary matrix with elements

$$
F_{m q}=\int d z \varphi_{m}(z) \hat{F} \varphi_{q}(z) .
$$

Note that $\hat{F} \varphi_{q}(z)$ is a solution to the parabolic equation (1) at range $r=L$ found for an initial condition $u(0, z)=\varphi_{q}(z)$. We have solved Eq. (10) using the code MMPE [32]. Numerical results presented below have been obtained for a carrier frequency $f=200 \mathrm{~Hz}$.

Associating a parameter $\mu$ with each function $\psi_{m}(z)$, where $\mu=\sum_{q} q\left|C_{m q}\right|^{2}$ is sum of intensity weighted numbers of eigenfunctions $\varphi_{q}$, we sort the Floquet modes in the order of increasing values of $\mu$. Therefore the eigenfunctions $\psi_{m}(z)$ with small $m$ describe waves localized at depths $z$ in the vicinity of the minimum of $U(0, z)$. The greater is the mode number $m$ the steeper are the grazing angles of waves forming the Floquet mode.

In order to link the structure of the Floquet mode with the classical phase space we shall consider the Husimi distribution function [2, 19]

$$
\begin{aligned}
& w_{m}(p, z)=\mid \frac{1}{\sqrt[4]{2 \pi \Delta_{z}^{2}}} \int d z^{\prime} \psi_{m}\left(z^{\prime}\right) \\
& \times \exp \left[i k p\left(z^{\prime}-z\right)-\frac{\left(z^{\prime}-z\right)^{2}}{4 \Delta_{z}^{2}}\right]^{2} \mid
\end{aligned}
$$

representing a projection of the wave field onto a Gaussian state with minimum uncertainty (coherent state) centered at a point $(p, z)$. Since the ray grazing angle $\theta$ with respect to the $r$-axis is related to the momentum by $\theta=\arctan p$ the distribution $w_{m}(p, z)$ may be interpreted as a local spectrum of the wave field smoothed over spatial and angular scales.

Figure 3 shows Husimi functions evaluated for six Floquet modes. These examples have been selected to demonstrate a variety of structures of Floquet modes in our environmental model. At each panel we have outlined borders of stable islands discernible in the central part of the phase portrait. Although the range variations of the potential $U$ are most pronounced at its minimum, the first Floquet eigenfunctions $\psi_{m}(z)$ are most closed to their unperturbed counterparts, i.e. to functions $\varphi_{m}(z)$ with the same numbers $m$. In the WKB approximation the Husimi function for any $\varphi_{m}(z)$ is a fuzzy version of a closed curve representing a period of an unperturbed ray whose Hamiltonian is equal to $E_{m}$. In Fig. 3 a we see an example of such a fuzzy curve. Here a Husimi function for $\psi_{10}(z)$ is plotted. It almost coincides with the unperturbed Husimi function for $\varphi_{10}(z)$ (not shown). The Husimi functions for the first 50 eigenfunctions $\psi_{m}(z)$ are localized within the central island. The fuzzy curves corresponding to $m$ approaching 50 repeat the shape of the border of the central island. This is clearly seen in Fig. $3 \mathrm{~b}$ where the Husimi function constructed for $\psi_{40}(z)$ is shown. 

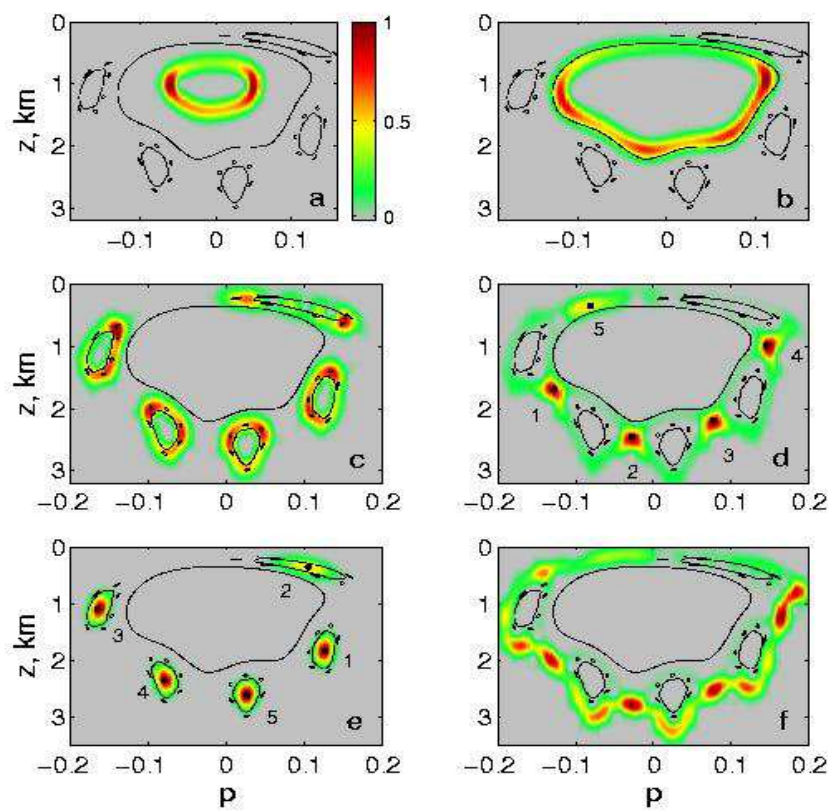

FIG. 3: Husimi distributions for the Floquet eigenfunctions $\psi_{10}(z)(\mathrm{a}), \psi_{40}(z)(\mathrm{b}), \psi_{66}(z)(\mathrm{c}), \psi_{77}(z)(\mathrm{d}), \psi_{88}(z)(\mathrm{e})$, and $\psi_{105}(z)$ (f). Parameter $\Delta_{z}=0.01 \mathrm{~km}$.
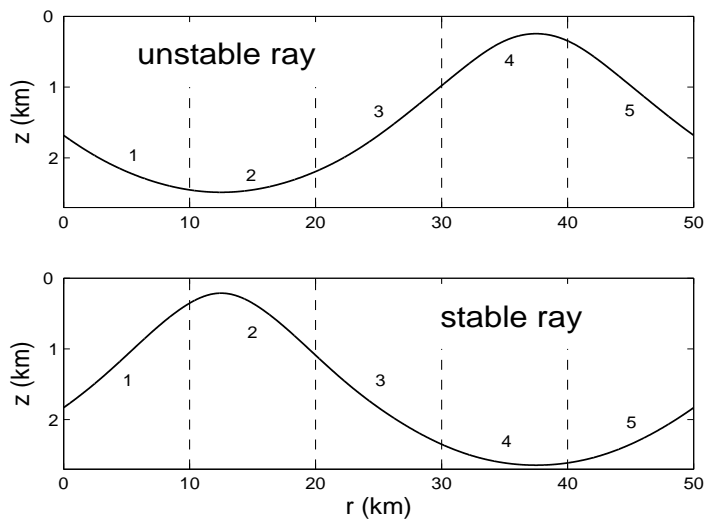

FIG. 4: Unstable (upper panel) and stable (lower panel) periodic rays with the period $50 \mathrm{~km}$.

Figures 3b-f demonstrate that the Husimi functions may have quite different structures. But there exists a remarkable common feature typical for all the first 140 Floquet modes considered in our numerical simulations: each distribution is localized within (i) the central stable island (Figs. 3a and b), (ii) within five subislands surrounding the central one (Fig. 3e), or (iii) within the chaotic sea (Figs. 3c, d, and f). Thus, the structure of Husimi functions correlates with the shape of stable islands. Roughly speaking, one may say that there are "chaotic" and "regular" Floquet modes whose Husimi functions are localized within areas of the phase space visited by predominantly chaotic or regular rays, respectively. Moreover, Fig. 3c presents a Husimi distribution localized outside the five sub-islands but in the vicinity of their borders. We conjecture that this is a finite wavelength analog of the stickiness to the borders of islands-surround-islands, i.e. the presence of such parts of the chaotic ray trajectory that correspond to the almost regular rotation of rays around the islands 34]. This occurs when, after wandering in the phase space, the trajectory approaches sub-islands and "sticks" to their borders for a fairly long time.
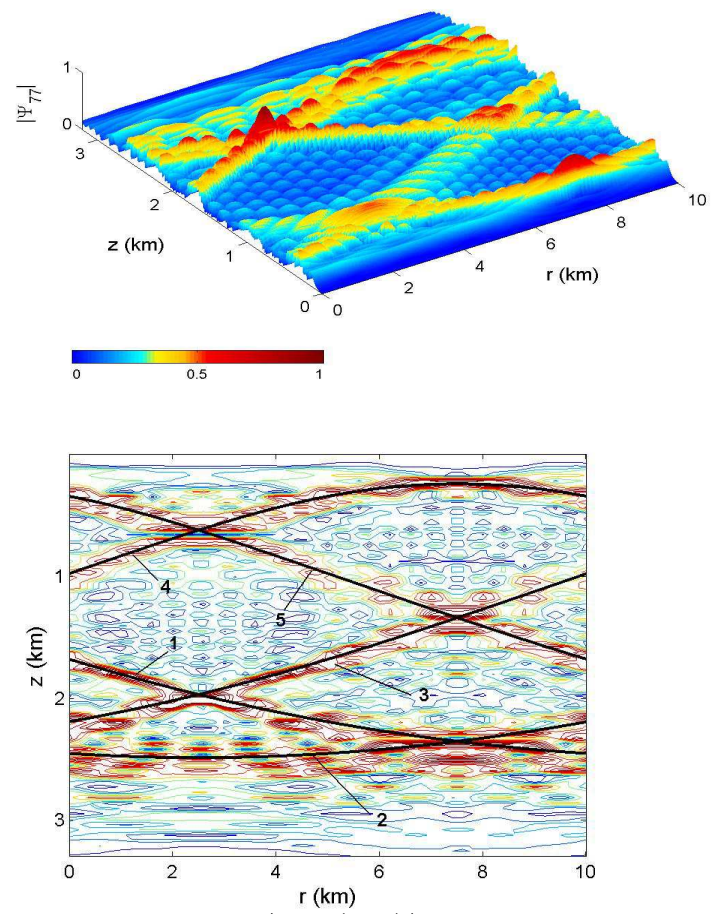

FIG. 5: Function $\left|\Psi_{77}(r, z)\right|$ at a period of its range-dependence. Five segments of the unstable ray from the upper panel of Fig. 4 are superimposed on the contour plot at the bottom.

Figures $3 \mathrm{~d}$ and 3e need more discussions. Each of them has five clearly seen maxima. In Fig. 3d the maxima are located within the chaotic sea, near the destroyed saddles, while in Fig. 3e they are located near the elliptic points of the sub-islands. We argue that these maxima are associated with trajectories of two periodic rays graphed in Fig. 4. Both rays have periods equal to $5 L=50 \mathrm{~km}$, while there are no other rays with the period of $50 \mathrm{~km}$. The ray shown in the upper panel of Fig. 4 belongs to the chaotic sea. The difference $|\delta z|$ between its coordinate and that of a ray with infinitesimally close starting parameters, on the average, grows as $\exp (\lambda r)$ with the Lyapunov exponent $\lambda=1 / 60 \mathrm{~km}^{-1}$. In contrast, the ray in the lower panel has a zero Lyapunov exponent, i.e. it is regular. Both rays have been divided into five $10 \mathrm{~km}$ segments. The segments connect the consecutive waveguide cross-sections at range points 
$r=n L$ chosen for the stroboscopic observation of both the ray structure and the Floquet modes (at these range they are presented by $\left.\psi_{m}(z)\right)$. Small black circles within red and yellow spots (corresponding to local maxima of Husimi function) in Fig. 3d indicate positions (in the phase plane) of starting points of five segments shown in the upper panel of Fig. 4. In agreement with our expectation these points lie at centers of five maxima of the Husimi function. The number of each segment is assigned to a corresponding circle and is indicated next to it in the figure. Similarly, the five maxima in Fig. 3e are associated with starting points of segments of the stable ray shown in the lower panel of Fig. 4. It is natural that the black circles depicting positions of these points are located in the centers of five stable sub-islands.
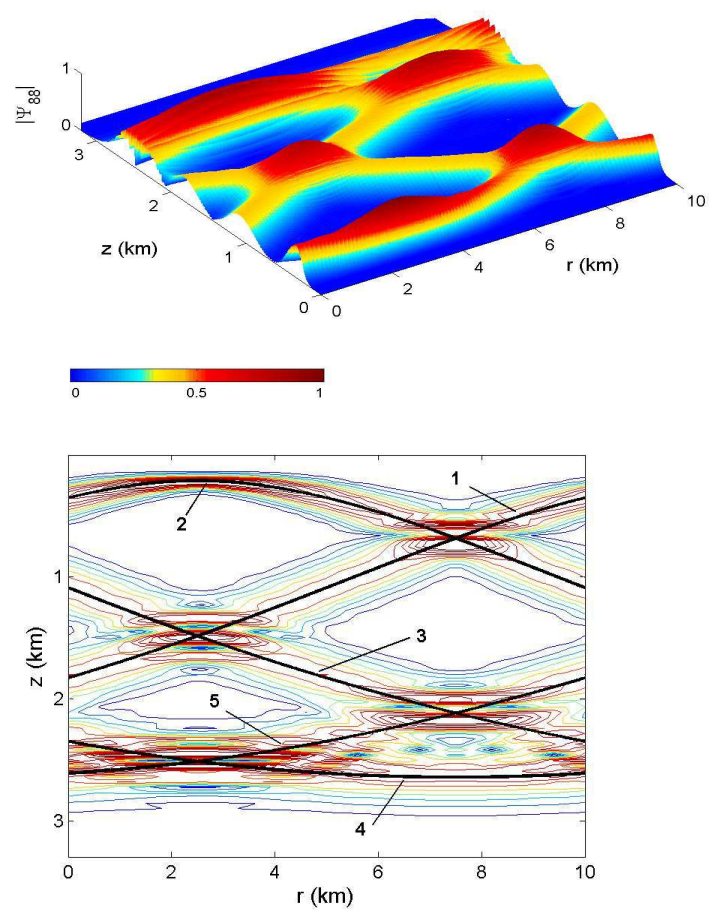

FIG. 6: The same as in Fig. 5 but for the Floquet mode $\Psi_{88}$. Five segments superimposed on the contour plot correspond to the stable ray from the lower panel of Fig. 4.

The above results suggest that the two periodic rays shown in Fig. 4 determine essentially the structure of the Floquet modes with $m=77$ and 88 . This conjecture is confirmed in Figs. 5 and 6 where we present distributions of intensities of these modes within a range interval $(0, L)$, i.e. over a spatial period of all the Floquet modes. Both functions $\left|\Psi_{77}(r, z)\right|$ and $\left|\Psi_{88}(r, z)\right|$, as well as $\left|\Psi_{66}(r, z)\right|$ shown in Fig. 7 are scaled to have absolute maxima equal to one. In Fig. 5 we see two representations of function $\left|\Psi_{77}(r, z)\right|$. In the lower panel five segments of the unstable ray from the upper panel of Fig. 4 are superimposed on the contour plot representing isolines of the intensity distribution. It is clearly seen that the intensity is high at the segments of the un- stable periodic ray and low elsewhere. So, the periodic unstable ray path scars the Floquet eigenfunction. This is an analog to the scarring phenomenon observed in different autonomous quantum systems [4, 5] (see also in [3]). In Fig. 6 it is seen that the 88-th Floquet mode localized within the five sub-islands also has relatively large amplitude in the vicinity of segments of the stable ray shown in the lower panel of Fig. 4. Moreover, practically all functions $\left|\Psi_{m}(r, z)\right|$ with $m>50$ display some pattern which may be associated with periodic rays similar to those shown in Figs. 5 and 6. We illustrate this statement in Fig. 7 for the Floquet mode with $m=66$.

Figures 5 and 6 suggest that the two Floquet modes may be considered as a superposition of five $10 \mathrm{~km}$ pieces of a wave beams propagating along the periodic unstable and stable rays, respectively. We conjecture that scars observed in intensity distributions of other modes, like that shown in Fig. 7, also may be associated with segments of some periodic rays. However, this issue needs a more detailed investigation.


FIG. 7: The same as in Fig. 5 but for the Floquet mode $\Psi_{66}$ and without ray segments superimposed on the contour plot.

The obtained results demonstrate that the scarring effect may be observed not only in autonomous quantum systems but also in driven ones described by the same equations as the waveguides with periodic rangedependencies. We presume that periodic rays determine the structures of the Floquet modes in approximately the same way as periodic orbits determine structures of the wave functions. The obtained results demonstrate that unstable chaotic rays can be considered as a reference direction for the corresponding wave packet propagation. 
This effect may be used for the transfer of information and communication.

This work was supported by the U.S. Navy Grants
N00014-97-1-0426, and N00014-02-1-0056, and by the Russian Foundation for Basic Research under Grant No. 03-02-17246.
[1] M. Gutzwiller, Chaos in classical and quantum mechanics (Springer-Verlag, New York, 1990).

[2] L. Reichl, The transition to chaos in conservative classical systems: quantum manifestations (Springer-Verlag, New York, 1992).

[3] H. Stökman, Quantum Chaos, An Introduction (Cambridge University Press, Cambridge, 1999).

[4] E. Heller, Phys. Rev. Let. 53, 1515 (1984).

[5] W. Bies, L. Kaplan, and E. Heller, Phys. Rev. E 64, 016204 (2001).

[6] Y. Takagaki and K. Ploog, Phys. Rev.B 70, 073304 (2004).

[7] E. Bogomolny, Physica D 31, 169 (1988).

[8] M. Berry, Proc. R. Soc. Lond. A 423, 219 (1989).

[9] M. Berry and J. Keating, Proc. R. Soc. Lond. A 437, 151 (1992).

[10] O. Agam and S. Fishman, Phys. Rev. Lett. 73, 806 (1994).

[11] S. Tomsovic and E. Heller, Phys. Rev. Lett. 70, 1405 (1993).

[12] S. Tomsovic and E. Heller, Phys. Rev. E 47, 282 (1993).

[13] P. F. Worcester, B. D. Cornuelle, M. A. Dzieciuch, W. H. Munk, M. Howe, A. Mercer, R. C. Spindel, J. A. Colosi, Metzger, T. Birdsall, et al., J. Acoust. Soc. Am. 105, 3185 (1999).

[14] M. Brown, J. Colosi, S. Tomsovic, A. Virovlyansky, M. Wolfson, and G. Zaslavsky, J. Acoust. Soc. Am. 113, 2533 (2003).

[15] F. Beron-Vera, M. Brown, J. Colosi, S. Tomsovic, A. Virovlyansky, M. Wolfson, and G. Zaslavsky, J. Acoust. Soc. Am. 114, 1226 (2003).

[16] S. Abdullaev and G. Zaslavsky, Usp. Fiz. Nauk 161, 1 (1991).

[17] S. Abdullaev, Chaos and dynamics of rays in waveguide media. Edited by G. Zaslavsky. (Gordon and Breach sci- ence publishers, New York, 1993).

[18] A. Virovlyansky, J. Acoust. Soc. Am. 108, 84 (2000).

[19] B. Sundaram and G. Zaslavsky, Chaos 9, 483 (1999).

[20] S. Abdullaev and G. Zaslavsky, Soviet Physics - JETP 53, 265 (1981).

[21] K. Smith, M. Brown, and F. Tappert, J. Acoust. Soc. Am. 91, 1939 (1992).

[22] I. Smirnov, A. Virovlyansky, and G. Zaslavsky, Phys. Rev. E 64, 036221 (2001).

[23] I. Smirnov, A. Virovlyansky, and G. Zaslavsky, Chaos 12, 617 (2002).

[24] I. Smirnov, A. Virovlyansky, and G. Zaslavsky, Chaos 14, 317 (2004).

[25] G. Zaslavsky and S. Abdullaev, Chaos 7, 182 (1997).

[26] F. Tappert, in Wave Propagation and Underwater Acoustics, edited by J. Keller and J. Papadakis (SpringerVerlag, Berlin; Heidelberg; New York, 1977), pp. 224284.

[27] L. Brekhovskikh and Y. Lysanov, Fundamentals of Ocean Acoustics (Springer-Verlag, Berlin, 1991).

[28] D. Palmer, M. Brown, F. Tappert, and H. Bezdek, Geophys. Res. Lett. 15, 569 (1988).

[29] M. Brown, F. Tappert, and G. Gon̂i, Wave motion 14, 93 (1991).

[30] D. Grempel, R. Prange, and S. Fishman, Phys. Rev. A 29, 1639 (1984).

[31] F. Jensen, W. Kuperman, M. Porter, and H. Schmidt, Computational Ocean Acoustics (AIP, Woodbury, New York, 1994).

[32] K. Smith, J. Comp. Acoust. 9, 243 (2001).

[33] V. Tatarskii, Sov. Phys. Uspekhi 26, 311 (1983), [Usp. Fiz. Nauk, v. 139, No. 4, pp.587-619 (1983)].

[34] G. Zaslavsky, M. Edelman, and B. Niyazov, Chaos 7, 159 (1997). 